\title{
Association of Ankle Brachial Index with Clinical Outcomes Following Percutaneous Coronary Intervention in Patients with Aortic Aneurysm
}

\author{
Yousuke Taniguchi, Kenichi Sakakura, Takunori Tsukui, Kei Yamamoto, Hiroyuki Jinnouchi, \\ Masaru Seguchi, Hiroshi Wada, Shin-ichi Momomura and Hideo Fujita
}

\begin{abstract}
:
Objective Since patients with thoracic aortic aneurysm (TAA)/abdominal aortic aneurysm (AAA) are often complicated with coronary artery disease, it is common for those patients to undergo percutaneous coronary intervention (PCI). The ankle brachial index (ABI) is usually measured in patients with TAA/AAA to screen the presence of peripheral arterial disease. The present study investigated the association between the ABI and clinical outcomes following PCI in patients with TAA/AAA.

Methods We divided 200 TAA/AAA patients who underwent PCI into a normal ABI group $(n=137)$ and an abnormal ABI group $(n=63)$ according to the ABI cut-off level of 1.00 . The primary endpoint was one-year major adverse cardiovascular events (MACE), defined as the composite of cardiovascular death, non-fetal myocardial infarction, stroke, target vessel revascularization, and hospitalization for heart failure.

Results Mean ABIs in the normal and abnormal ABI groups were $1.12 \pm 0.09$ and $0.86 \pm 0.11$, respectively (p $<0.01$ ). Kaplan-Meier curves showed MACE were more frequent in the abnormal ABI group than in the normal ABI group ( $\mathrm{p}=0.01$ ). A multivariate Cox hazard analysis revealed that an abnormal ABI was significantly associated with 1-year MACE (vs. ABI $\geq 1.0$ : HR 3.02, 95\% confidence interval 1.00-9.08, $\mathrm{p}=0.049$ ).

Conclusion Among patients with TAA/AAA who underwent PCI, abnormal ABI was significantly associated with 1-year MACE, suggesting the utility of the ABI measurement in this high-risk population.
\end{abstract}

Key words: percutaneous coronary interventions, aortic aneurysm, ankle brachial index

(Intern Med 60: 2733-2740, 2021)

(DOI: 10.2169/internalmedicine.6816-20)

\section{Introduction}

Thoracic aortic aneurysm (TAA) and abdominal aortic aneurysm (AAA) are major vascular diseases induced by systemic atherosclerosis. TAA and AAA are related to increased morbidity and mortality rates, especially due to the rupture of TAA/AAA $(1,2)$. Since the mortality of TAA/ AAA rupture is still very high, open surgery or endovascular aortic repair should be performed before rupture occurs (2-4). Furthermore, patients with TAA/AAA often have coronary artery disease (CAD), which results in an even greater mortality rate $(5,6)$. Therefore, it is not uncommon for patients with TAA/AAA to undergo percutaneous coro- nary intervention $(\mathrm{PCI})(6,7)$.

The ankle brachial index (ABI) has been reported as an indicator of clinical outcomes in patients who underwent PCI (8-10). ABI is widely performed in patients with TAA/ AAA, as TAA/AAA is often complicated with peripheral artery disease (11). Thus, patients with TAA/AAA who undergo PCI usually have available data on their ABI, regardless of the presence of peripheral artery disease. However, the significance of the ABI in TAA/AAA patients who undergo PCI has not been established, as few reports have described the association between the ABI and clinical outcomes in TAA/AAA patients with CAD. We hypothesized that the ABI could be a simple and useful predictor of major adverse cardiovascular events (MACE) in TAA/AAA pa- 
tients who underwent PCI. The present study investigated the association between the ABI and 1-year MACE in TAA/ AAA patients who underwent PCI.

\section{Materials and Methods}

\section{Study design}

We reviewed consecutive PCI cases in our medical center between January 2016 and December 2018. The inclusion criteria were 1) patients who underwent PCI and 2) patients who had TAA/AAA at the day of PCI. The exclusion criteria were 1) PCI to graft lesions, and 2) a lack of ABI data (within one year before or one month after the index PCI). We divided the final study population into two groups according to the ABI. Cases with a preserved ABI (ABI $\geq$ 1.00) were considered the normal ABI group, while those with borderline $(0.90$ to $<1.00)$ or decreased values $(<0.90)$ were defined as the abnormal ABI group $(8,12,13)$.

The primary endpoint was MACE at one year after PCI. MACE were defined as the composite of cardiovascular death, non-fetal myocardial infarction, non-fatal stroke, any target vessel revascularization, and hospitalization for acute de-compensated heart failure (ADHF). Non-fatal myocardial infarction was defined according to the universal definition (14). The index day was set as the day of PCI.

All therapeutic strategies related to TAA/AAA and CAD were considered and discussed with cardiovascular surgeons, and we decided not only the indication but also the order and timing of treatments.

\section{$\mathrm{PCl}$ procedure}

We decided PCI indication and strategy at our daily conference except emergent PCI cases. In elective PCI for patients with TAA, we consult cardiovascular surgeons about the possibility of open thoracic surgery with simultaneous CABG, and about the timing of PCI (before or after surgery). In elective PCI for patients with AAA, we consult cardiovascular surgeons about the timing of PCI (before surgery or after surgery). The high-risk or complex cases in the present study were discussed at a weekly heart team conference consisting of cardiologists and cardiovascular surgeons. The radial artery was most frequently used, followed by the brachial artery, with the femoral artery being used the least, because of TAA/AAA. We maintained an activated clotting time of at least 250 seconds. The selection of devices was left to the discretion of each interventional cardiologist. We tried to avoid the insertion of mechanical support devices, such as an intra-aortic balloon pump (IABP) or veno-arterial extracorporeal membrane oxygenation (VA-ECMO). Regarding complications, peri-procedural myocardial infarction in non-emergent cases was defined as cases where the creatine kinase values the day after PCI increased to more than twice the upper normal limit (15).

\section{Definitions}

TAA/AAA was defined in cases with a history of open or endovascular surgeries or maximum aortic diameter $\geq 45 \mathrm{~mm}$ for TAA and $\geq 30 \mathrm{~mm}$ for AAA (16). Hypertension was defined as receiving treatment for hypertension before admission $(17,18)$. Dyslipidemia was defined as total cholesterol $>220 \mathrm{mg} / \mathrm{dL}$, low-density lipoprotein cholesterol (LDL) > $140 \mathrm{mg} / \mathrm{dL}$, or medical treatment for dyslipidemia before admission (17-19). Diabetes mellitus (DM) was defined as hemoglobin A1c (HbA1c) >6.5\% [national glycol-hemoglobin standardization program (NGSP) value] or medical treatment for $\operatorname{DM}(17,19,20)$. The estimated glomerular filtration rate (eGFR) was calculated by the modification of diet in renal disease (MDRD) method, adjusted for a Japanese population $(21,22)$. Anemia was determined by the World Health Organization (WHO) criteria as a hemoglobin $(\mathrm{Hb})$ value < $13 \mathrm{~g} / \mathrm{dL}$ in men and $<12 \mathrm{~g} / \mathrm{dL}$ in women $(23,24)$. ST elevation myocardial infarction (STEMI), non-ST elevation myocardial infarction (NSTEMI), and unstable angina pectoris (UAP) were defined according to the universal definitions (14). Acute coronary syndrome (ACS) was defined as a composite of STEMI, NSTEMI, and UAP.

\section{Statistical analyses}

Categorical data were presented as number and percentage, and continuous data were presented as the mean \pm standard deviation (SD). Normally distributed continuous variables were compared using an unpaired Student's $t$-test. Other continuous variables were compared using the MannWhitney $U$-test. Categorical data were compared using the chi-square test or Fisher's exact test. A Kaplan-Meier survival analysis was performed to compare 1-year MACE between the normal and abnormal ABI groups, and difference were assessed by the log-rank test. We also performed a multivariate Cox hazard analysis (stepwise method: backward elimination using likelihood ratio) to examine the associations between an abnormal ABI and 1-year MACE after controlling confounding factors. In this model, MACE were used as a dependent variable, and an abnormal ABI was the independent variable. We applied confounding factors, defined as variables with a $\mathrm{P}$ value $<0.10$ in the univariate analyses. Although there were significant differences between the normal and abnormal ABI groups, variables that had missing values were not included in the multivariate analysis. Hazard ratios (HRs) and 95\% confidence intervals (CIs) were calculated. A p value $<0.05$ was considered statistically significant. All analyses were performed with IBM SPSS statistics version 25 (Chicago, USA).

\section{Results}

We screened 2,387 consecutive PCI cases encountered at our center between January 2016 and December 2018. Of those, 211 were diagnosed with or treated for TAA/AAA. Eleven cases were excluded because of PCI to a saphenous 


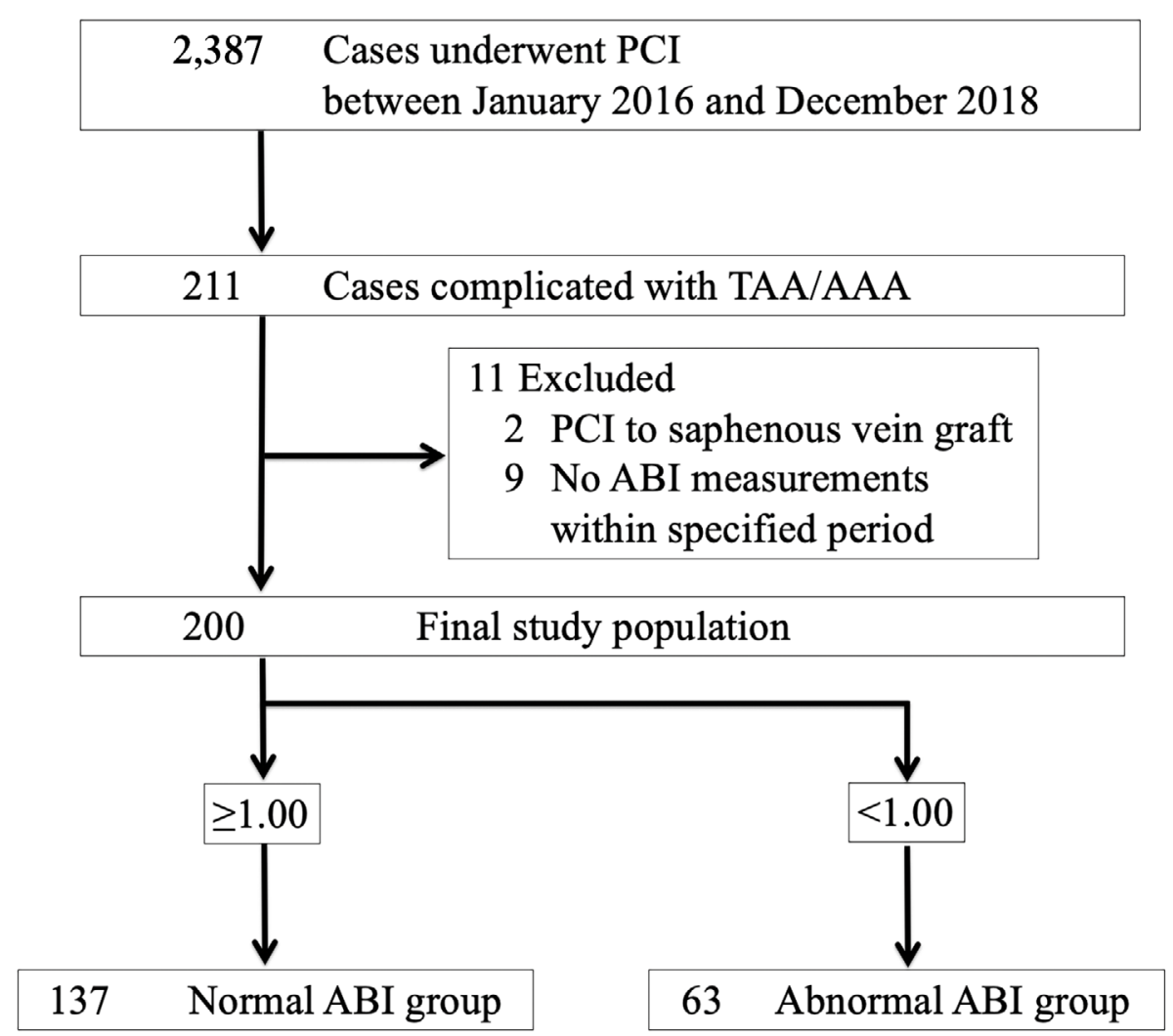

Figure 1. Flowchart of patient selection and how patients were divided into two groups. AAA: abdominal aortic aneurysm, ABI: ankle brachial index, PCI: percutaneous coronary intervention, TAA: thoracic aortic aneurysm

vein graft or a lack of $\mathrm{ABI}$ data (within one year before or one month after the index PCI). A total of 200 PCI cases were thus included as the final study population and divided into the normal ABI group $(n=137)$ and abnormal ABI group (n=63) (Fig. 1).

Table 1 shows the comparison of the baseline characteristics between the two groups. As defined, the ABI was significantly higher in the normal ABI group than in the abnormal group $(1.12 \pm 0.09$ vs. $0.86 \pm 0.11, \mathrm{p}<0.01)$. The eGFR was also higher in the normal ABI group than in the abnormal group $\left(59.5 \pm 20.1 \mathrm{~mL} / \mathrm{min} / 1.73 \mathrm{~m}^{2}\right.$ vs. $53.4 \pm 23.6 \mathrm{~mL} /$ $\left.\min / 1.73 \mathrm{~m}^{2}, \mathrm{p}=0.04\right)$. BNP values were lower in the normal ABI group than in the abnormal ABI group $(211 \pm 409 \mathrm{pg} /$ $\mathrm{mL}$ vs. $336 \pm 480 \mathrm{pg} / \mathrm{mL}, \mathrm{p}=0.01)$. The use of diuretics at discharge were more frequently observed in the abnormal ABI group than in the normal ABI group $(25.5 \%$ vs. $47.6 \%$, $\mathrm{p}<0.01)$.

Table 2 shows the comparison of the procedural characteristics between the two groups. The prevalence of ACS was lower in the normal ABI group than in the abnormal ABI group (29.2\% vs. $46.0 \%, p=0.03)$. VA-ECMO was more frequently inserted in the abnormal $\mathrm{ABI}$ group than in the normal ABI group (0\% vs. 6.3\%, p<0.01).

We constructed Kaplan-Meier curves, and 1-year MACE were significantly better in the normal ABI group than in the abnormal ABI group (Fig. 2) $(\mathrm{p}=0.01)$. We also performed Fisher's exact tests regarding individual outcomes, including MACE, between the two groups (Table 3). The causes of five cardiovascular deaths were as follows: acute myocardial infarction in one, $\mathrm{HF}$ in one, sudden death just after thoracic aortic replacement (without rupture) in one, and sudden death due to unknown causes in two.

Table 4 shows the results of a multivariate Cox hazard analysis. Although the initial model included an abnormal ABI, history of hypertension, eGFR $<30 \mathrm{~mL} / \mathrm{min} / 1.73 \mathrm{~m}^{2}$, moderate or severe AR, diuretics user, insulin user, ACS, multi-vessel disease, and VA-ECMO use, the final model included only 5 variables (ABI, multi-vessel disease, ACS, moderate/severe AR, and insulin user) according to the stepwise method (backward elimination and likelihood ratio). An abnormal ABI <1.0 (HR 3.02, 95\% CI 1.00-9.08, p=0.049) was significantly associated with 1-year MACE after controlling for confounding factors.

\section{Discussion}

We divided 200 patients with TAA/AAA who underwent PCI into the normal ABI group $(n=137)$ and abnormal ABI group ( $n=63$ ). The Kaplan-Meier curves showed that the incidence of MACE was higher in the abnormal ABI group than in the normal ABI group. The multivariate Cox hazard analysis revealed that an abnormal ABI was significantly associated with 1-year MACE (HR 3.02, 95\% CI 1.00-9.08, p $=0.049$ ) after controlling for confounding factors. Several re- 
Table 1. Comparison of Patient Characteristics between the Normal ABI Group and Abnormal ABI Group.

\begin{tabular}{|c|c|c|c|c|}
\hline & $\begin{array}{l}\text { Overall } \\
(\mathrm{n}=200)\end{array}$ & $\begin{array}{l}\text { Normal ABI group } \\
(\mathrm{n}=137)\end{array}$ & $\begin{array}{c}\text { Abnormal ABI group } \\
(\mathrm{n}=63)\end{array}$ & $\mathrm{p}$ value \\
\hline Age (years old) & $74.3 \pm 7.7$ & $74.4 \pm 8.0$ & $74.1 \pm 7.3$ & 0.79 \\
\hline Female gender (No.) (\%) & $26(13.0)$ & $16(11.7)$ & $10(15.9)$ & 0.50 \\
\hline Body mass index $\left(\mathrm{kg} / \mathrm{m}^{2}\right)$ & $23.1 \pm 3.6$ & $23.2 \pm 3.9$ & $22.7 \pm 2.7$ & 0.39 \\
\hline Smoker (No.) (\%) & $160(80.0)$ & 109 (79.6) & $51(81.0)$ & 1.00 \\
\hline Hypertension (No.) (\%) & $181(90.5)$ & $128(93.4)$ & $53(84.1)$ & 0.07 \\
\hline Dyslipidemia (No.) (\%) & $167(83.5)$ & $117(85.4)$ & $50(79.4)$ & 0.31 \\
\hline Diabetes mellitus (No.) (\%) & $54(27.0)$ & $38(27.7)$ & $16(25.4)$ & 0.86 \\
\hline History of hospitalization of ADHF (No.) (\%) & $8(4.0)$ & $5(3.6)$ & $3(4.8)$ & 0.71 \\
\hline AAA (No.) (\%) & $174(87.0)$ & $120(87.6)$ & $54(85.7)$ & 0.82 \\
\hline TAA (No.) (\%) & $51(25.5)$ & $36(26.3)$ & $15(23.8)$ & 0.86 \\
\hline Post intervention to aneurysms (No.) (\%) & $71(35.5)$ & $51(37.2)$ & $20(31.7)$ & 0.53 \\
\hline $\mathrm{ABI}$ & $1.04 \pm 0.15$ & $1.12 \pm 0.09$ & $0.86 \pm 0.11$ & $<0.01$ \\
\hline \multicolumn{5}{|l|}{ Laboratory data } \\
\hline HbA1c (NGSP) (\%) (n=198) & $6.1 \pm 0.9$ & $6.1 \pm 0.6$ & $6.2 \pm 1.3$ & 0.23 \\
\hline Creatinine (mg/dL) & $1.30 \pm 1.47$ & $1.17 \pm 1.12$ & $1.58 \pm 2.01$ & 0.09 \\
\hline eGFR $\left(\mathrm{mL} / \mathrm{min} / 1.73 \mathrm{~m}^{2}\right)$ & $57.6 \pm 21.4$ & $59.5 \pm 20.1$ & $53.4 \pm 23.6$ & 0.04 \\
\hline LDL cholesterol $(\mathrm{mg} / \mathrm{dL})(\mathrm{n}=191)$ & $94 \pm 28$ & $97 \pm 29$ & $89 \pm 25$ & 0.16 \\
\hline Hemoglobin (g/dL) & $12.8 \pm 2.9$ & $13.0 \pm 3.2$ & $12.4 \pm 1.7$ & 0.13 \\
\hline Anemia (WHO criteria) (No.) (\%) & $109(54.5)$ & $74(54.0)$ & $35(55.6)$ & 0.88 \\
\hline Prothrombin time-INR $(\mathrm{n}=186)$ & $1.08 \pm 0.19$ & $1.06 \pm 0.15$ & $1.14 \pm 0.24$ & 0.02 \\
\hline $\operatorname{APTT}(\mathrm{s})(\mathrm{n}=190)$ & $39.6 \pm 28.7$ & $36.6 \pm 22.0$ & $45.9 \pm 38.7$ & 0.01 \\
\hline$D$-dimer $(n=123)$ & $4.16 \pm 7.32$ & $4.39 \pm 8.73$ & $3.70 \pm 3.23$ & 0.63 \\
\hline $\mathrm{BNP}(\mathrm{pg} / \mathrm{mL})(\mathrm{n}=189)$ & $251 \pm 436$ & $211 \pm 409$ & $336 \pm 480$ & 0.01 \\
\hline \multicolumn{5}{|l|}{ Echo cardiography } \\
\hline $\begin{array}{l}\text { Left ventricular EF } \\
\text { (modified Simpson's methods) }(\%)(n=124)\end{array}$ & $52.6 \pm 17.5$ & $54.5 \pm 15.8$ & $48.0 \pm 20.6$ & 0.18 \\
\hline Moderate/severe AR (No.) (\%) & $8(4.0)$ & $8(5.8)$ & $0(0.0)$ & 0.06 \\
\hline \multicolumn{5}{|l|}{ Medication at discharge } \\
\hline Aspirin (No.) (\%) & $200(100)$ & $137(100)$ & $63(100)$ & - \\
\hline P2 $\mathrm{Y}_{12}$ inhibitors (No.) (\%) & & & & 0.13 \\
\hline Clopidogrel & $147(73.5)$ & $100(73.0)$ & $47(74.6)$ & \\
\hline Prasugrel & $49(24.5)$ & $36(26.3)$ & $13(20.6)$ & \\
\hline Oral anti-coagulants (No.) (\%) & $29(14.5)$ & $19(13.9)$ & $10(15.9)$ & 0.83 \\
\hline Statins (No.) (\%) & $194(97.0)$ & $132(96.4)$ & $62(98.4)$ & 0.67 \\
\hline ACEI and/or ARB (No.) (\%) & $137(68.5)$ & $92(67.2)$ & 45 (71.4) & 0.62 \\
\hline$\beta$ blockers (No.) (\%) & $157(78.5)$ & $105(76.6)$ & $52(82.5)$ & 0.46 \\
\hline Diuretics (No.) (\%) & $65(32.5)$ & $35(25.5)$ & $30(47.6)$ & $<0.01$ \\
\hline Oral hypoglycemic agents (No.) (\%) & $32(16.0)$ & $19(13.9)$ & $13(20.6)$ & 0.30 \\
\hline Insulin (No.) (\%) & $4(2.0)$ & $1(0.7)$ & $3(4.8)$ & 0.09 \\
\hline
\end{tabular}

WHO criteria: the hemoglobin of the male $<13 \mathrm{~g} / \mathrm{dL}$ and the female $<12 \mathrm{~g} / \mathrm{dL}$

ABI: ankle brachial index, ADHF: acute decompensated heart failure, AAA: abdominal aortic aneurysm, TAA: thoracic aortic aneurysm, HbA1c: hemoglobin A1c, NGSP: national glycohemoglobin standardization program, eGFR: estimated glomerular filtration rate, LDL: low density lipoprotein, WHO: World Health Organization, INR: international normalized ratio, APTT: activated partial thromboplastin time, BNP: brain natriuretic peptide, EF: ejection fraction, AR: aortic valve regurgitation, ACEI: angiotensin converting enzyme inhibitors, ARB: angiotensin II receptor blockers

ports concerning members of the general population who underwent PCI have described the relationship between the ABI and MACE $(8-10,12,25)$. Sasaki et al. (2020) reported that a lower ABI was a good predictor of MACE after drugeluting stent implantation (9). Furthermore, Nishimura et al. (2017) mentioned that an ABI $<1.00$ predicted future inci- dent of hospitalization for ADHF (25). However, our study, which focused on TAA/AAA cases, is unique in elucidating the relationship between ABI and MACE.

Regarding why an abnormal ABI was associated with MACE after PCI in patients with TAA/AAA, when we considered each parameter of MACE, the incidence of hospitali- 
Table 2. Comparison of Interventions between the Normal ABI Group and the Abnormal ABI Group.

\begin{tabular}{|c|c|c|c|c|}
\hline & $\begin{array}{l}\text { Overall } \\
(\mathrm{n}=200)\end{array}$ & $\begin{array}{l}\text { Normal ABI group } \\
\quad(n=137)\end{array}$ & $\begin{array}{c}\text { Abnormal ABI group } \\
(n=63)\end{array}$ & $\mathrm{p}$ value \\
\hline Emergent PCI (No.) (\%) & $37(18.5)$ & $23(16.8)$ & $14(22.2)$ & 0.43 \\
\hline Acute coronary syndrome (No.) (\%) & $69(34.5)$ & $40(29.2)$ & $29(46.0)$ & 0.03 \\
\hline Approach site & & & & 0.23 \\
\hline Right radial or brachial (No.) (\%) & $114(57.0)$ & $79(57.7)$ & $35(55.6)$ & \\
\hline Left radial or brachial (No.) (\%) & $48(24.0)$ & $36(26.3)$ & $12(19.0)$ & \\
\hline Femoral (No.) (\%) & $38(19.0)$ & $22(16.1)$ & $16(25.4)$ & \\
\hline Sheath size (No.) (\%) & & & & 0.20 \\
\hline $6 \mathrm{Fr}$ & $104(52.0)$ & $77(56.2)$ & $27(42.9)$ & \\
\hline $7 \mathrm{Fr}$ & $89(44.5)$ & $56(40.9)$ & $33(52.4)$ & \\
\hline$\geq 8 \mathrm{Fr}$ & $7(3.5)$ & $4(2.9)$ & $3(4.8)$ & \\
\hline Culprit lesions & & & & 0.26 \\
\hline Right coronary (No.) (\%) & $77(38.5)$ & $53(38.7)$ & $24(38.1)$ & \\
\hline Left main (No.) (\%) & $10(5.0)$ & $4(2.9)$ & $6(9.5)$ & \\
\hline Left anterior descending (No.) (\%) & $86(43.0)$ & $61(44.5)$ & $25(39.7)$ & \\
\hline Left circumflex (No.) (\%) & $27(13.5)$ & $19(13.9)$ & $8(12.7)$ & \\
\hline Multi-vessel disease (No.) (\%) & $110(55.0)$ & $69(50.4)$ & $41(65.1)$ & 0.07 \\
\hline \multicolumn{5}{|l|}{ Lesion types and strategies } \\
\hline In-stent restenosis (No.) (\%) & $13(6.5)$ & $6(4.4)$ & $7(11.1)$ & 0.12 \\
\hline Chronic total obstruction (No.) (\%) & $20(10.0)$ & $15(10.9)$ & $5(7.9)$ & 0.62 \\
\hline Rotablation (No.) (\%) & $24(12.0)$ & $16(11.7)$ & $8(12.7)$ & 0.82 \\
\hline Jailed micro-catheter (No.) (\%) & $3(1.5)$ & $2(1.5)$ & $1(1.6)$ & 1.00 \\
\hline Post optimized technique (No.) (\%) & $15(7.5)$ & $8(5.8)$ & $7(11.1)$ & 0.25 \\
\hline Kissing balloon technique (No.) (\%) & $5(2.5)$ & $3(2.2)$ & $2(3.2)$ & 0.65 \\
\hline Micro-catheters (No.) (\%) & $66(33.0)$ & $41(29.9)$ & $25(39.7)$ & 0.20 \\
\hline Extension catheters (No.) (\%) & $30(15.0)$ & $24(17.5)$ & $6(9.5)$ & 0.20 \\
\hline POBA alone (No.) (\%) & $8(4.0)$ & $3(2.2)$ & $5(7.9)$ & 0.11 \\
\hline DCB alone (No.) (\%) & $16(8.0)$ & $10(7.3)$ & $6(9.5)$ & 0.58 \\
\hline Stenting (No.) (\%) & $177(88.5)$ & $124(90.5)$ & $53(84.1)$ & 0.23 \\
\hline Drug eluting stents $(\mathrm{n}=177)($ No.) $(\%)$ & $161(91.0)$ & $112(90.3)$ & $49(92.5)$ & 0.78 \\
\hline \multicolumn{5}{|l|}{ Mechanical support devices } \\
\hline IABP (No.) (\%) & $5(2.5)$ & $2(1.5)$ & $3(4.8)$ & 0.18 \\
\hline VA-ECMO (No.) (\%) & $4(2.0)$ & $0(0)$ & $4(6.3)$ & $<0.01$ \\
\hline Successful PCI (No.) (\%) & $198(99.0)$ & $136(99.3)$ & $62(98.4)$ & 0.53 \\
\hline Contrast volume $(\mathrm{mL})$ & $112 \pm 58$ & $112 \pm 49$ & $114 \pm 76$ & 0.46 \\
\hline Exposure time (min) & $24 \pm 18$ & $23 \pm 16$ & $25 \pm 20$ & 0.93 \\
\hline Peri-procedural MI (No.) (\%) & $8(4.0)$ & $5(3.6)$ & $3(4.8)$ & 0.71 \\
\hline
\end{tabular}

ABI: ankle brachial index, PCI: percutaneous coronary intervention, POBA: plane old balloon angioplasty, DCB: drug coated balloon, IABP: intra-aortic balloon pumping, VA-ECMO: veno-arterial extracorporeal membrane oxygenation, MI: myocardial infarction

zation caused by ADHF tended to be greater in the abnormal ABI group than in the normal ABI group, albeit without reaching statistical significance $(2.9 \%$ vs. $7.9 \%, \mathrm{p}=0.14)$. Several groups reported the relationship between an abnormal ABI and ADHF (12, 25). Gupta et al. (2014) described the significant association between an abnormal ABI and HF in a middle-age community cohort $(n=1,315)$ and speculated that vascular stiffness and atherosclerotic microvascular dysfunction might provoke $\mathrm{HF}$ in patients with an abnormal ABI (12). An impaired vascular compliance was shown to be associated with a low ABI (26). Vascular stiffness increases the left ventricular afterload, which may impair the coronary blood flow and induce myocardial ischemia, even without significant coronary artery stenosis (27).
Furthermore, an abnormal ABI may indicate systemic atherosclerosis or poly-vascular disease (8). Indeed, CAD is often complicated with peripheral arterial diseases or cerebral vascular disease (28), which have been associated with MACE after PCI (29). Since the presence of TAA/AAA and severe CAD was an inclusion criterion in the present study, all study patients already had at least two major atherosclerotic risk factors. In the present study population, abnormal ABI referred to the presence of another atherosclerotic risk factor, suggesting more advanced atherosclerosis in the abnormal ABI group than in the normal ABI group. Therefore, the abnormal ABI cases was associated with one-year MACE.

Our results suggest that $\mathrm{ABI}$ measurement is useful in pa- 


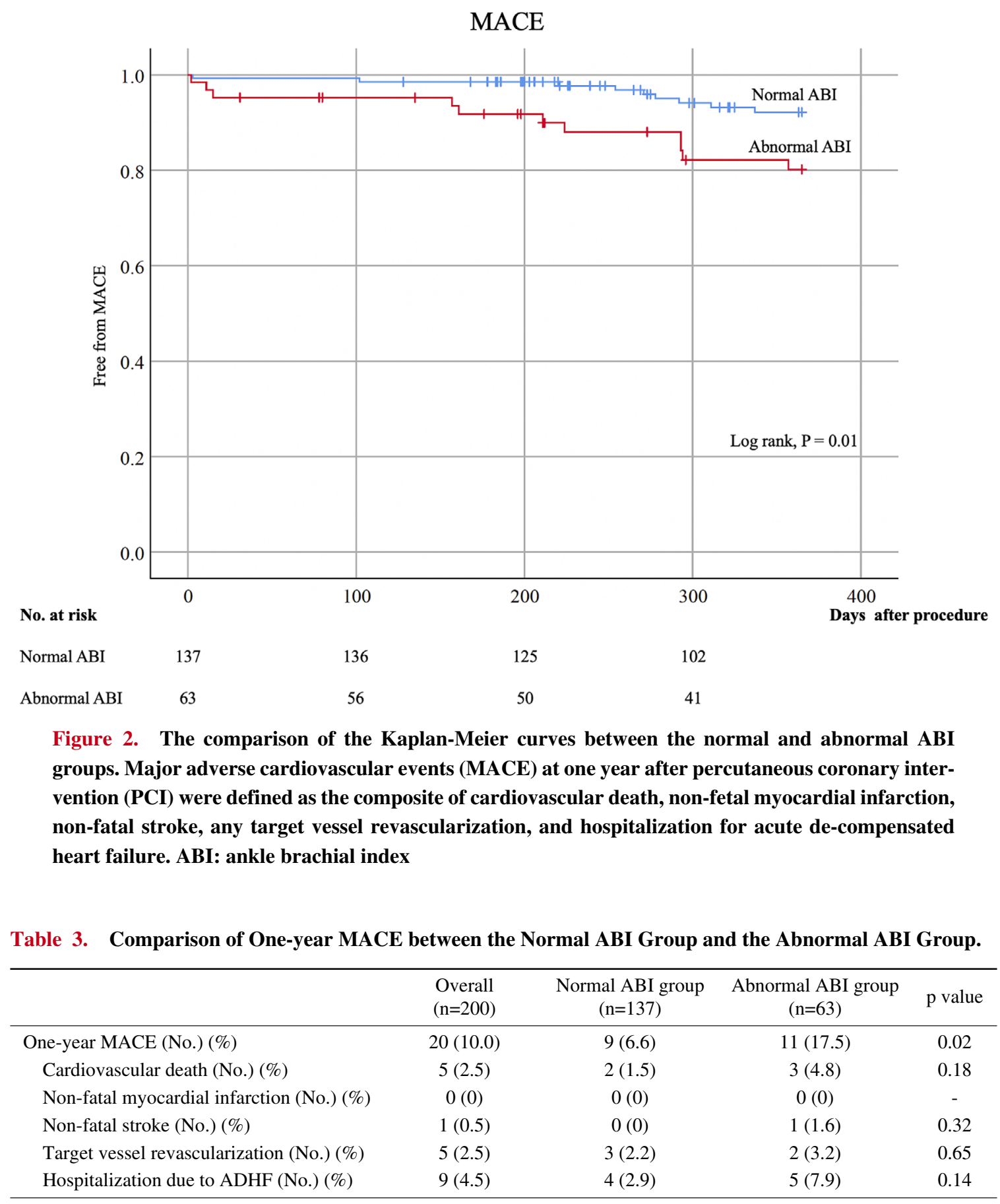

ABI: ankle brachial index, ADHF: acute decompensated heart failure, MACE: major adverse cardiovascular event (composite of cardiovascular death, non-fatal myocardial infarction, non-fatal stroke, target vessel revascularization, and hospitalization due to ADHF)

tients with TAA/AAA who undergo PCI, as the ABI can be a marker predicting the clinical outcomes after PCI. Furthermore, an exercise stress test, which is an established predictor of clinical outcomes after PCI (30), tends to be avoided among patients with TAA/AAA due to concerns about the rupture of TAA/AAA. The ABI thus has a great advantage over exercise stress tests with regard to its non-invasiveness.

The present study revealed that ACS (HR 3.90, 95\% CI 1.48-10.22, $\mathrm{p}=0.006$ ), moderate/severe AR (HR 8.31, 95\% CI 1.96-35.29, p=0.004) and insulin users (HR 8.88, 95\% CI 2.14-36.87, $\mathrm{p}=0.003$ ) were associated with 1-year MACE. It is well known that patients with ACS have worse clinical outcomes than those with stable angina (31). Although moderate/severe AR has been shown to be associated with MACE, there have been few reports describing the relationship between AR and MACE after PCI. However, severe AR can be a cause of HF. Since MACE included hospitalization for ADHF in the present study, AR might be associated with MACE. Insulin use was also associated with MACE. It is known that, among patients after PCI, the incidence of MACE is greater in insulin users than in nonusers (32).

Several limitations associated with the present study warrant mention. Since this study was a retrospective study, 
Table 4. Multivariate COX Hazard Analysis.

\begin{tabular}{lccc}
\hline $\begin{array}{c}\text { Independent variables for } \\
\text { "one-year MACE” }\end{array}$ & HR & 95\% CI & p value \\
\hline Peri-procedural ABI $<1.0$ & 3.02 & $1.00-9.08$ & 0.049 \\
Multi-vessel disease & 2.60 & $0.85-7.98$ & 0.096 \\
Acute coronary syndrome & 3.90 & $1.48-10.22$ & 0.006 \\
Moderate/severe AR & 8.31 & $1.96-35.29$ & 0.004 \\
Insulin users & 8.88 & $2.14-36.87$ & 0.003 \\
\hline
\end{tabular}

Covariates were $\mathrm{ABI}<1.0$, history of hypertension, eGFR $<30 \mathrm{~mL} /$ $\min / 1.73 \mathrm{~m}^{2}$, moderate or severe AR, diuretics user, insulin user, acute coronary syndrome, multi-vessel disease, and VA-ECMO use.

Analysis was performed by stepwise method (backward elimination and likelihood ratio) and fifth step was final.

ABI: ankle brachial index, AR: aortic valve regurgitation, MACE: major adverse cardiovascular event (composite of cardiovascular death, non-fatal myocardial infarction, non-fatal stroke, target vessel revascularization, and hospitalization due to ADHF)

there was a potential selection bias. Because the sample size was limited, the statistical analysis carried an inherent risk of beta error (33). Since the number of 1-year MACE was small $(n=20,10.0 \%$ of the sample volume), the number of independent variables was limited in the multivariate Cox hazard analysis. Further studies including a sufficient study population are warranted to confirm the relationship between an abnormal ABI and clinical outcomes after PCI among patients with TAA/AAA.

\section{Conclusion}

Among patients with TAA/AAA who underwent PCI, an abnormal ABI was significantly associated with 1-year MACE, suggesting the utility of measuring the ABI in this high-risk population.

\section{Author's disclosure of potential Conflicts of Interest (COI).}

Kenichi Sakakura: Employment, Boston Scientific and Abbott Vascular; Honoraria, Abbott Vascular, Boston Scientific, Medtronic Cardiovascular, Terumo, OrbusNeich, Japan Lifeline and NIPRO. Hideo Fujita: Employment, Mehergen Group Holdings.

\section{References}

1. Vallabhaneni SR. Final follow-up of the Multicentre Aneurysm Screening Study (MASS) randomized trial of abdominal aortic aneurysm screening. Br J Surg 99: 1649-1656, 2012.

2. Guo MH, Appoo JJ, Saczkowski R, et al. Association of mortality and acute aortic events with ascending aortic aneurysm: a systematic review and meta-analysis. JAMA Netw Open 1: e181281, 2018.

3. Isselbacher EM, Lino Cardenas CL, Lindsay ME. Hereditary influence in thoracic aortic aneurysm and dissection. Circulation 133: 2516-2528, 2016.

4. Tchana-Sato V, Sakalihasan N, Defraigne JO. Ruptured abdominal aortic aneurysm. Rev Med Liege 73: 296-299, 2018.

5. Daimon M, Miyata H, Motomura N, et al. Outcomes of thoracic aortic surgery in patients with coronary artery disease-based on the Japan Adult Cardiovascular Surgery Database. Circ J 83: 978-984,
2019.

6. Holda MK, Iwaszczuk P, Wszolek K, et al. Coexistence and management of abdominal aortic aneurysm and coronary artery disease. Cardiol J 27: 384-393, 2020.

7. Girardi LN, Rabotnikov Y, Avgerinos DV. Preoperative percutaneous coronary intervention in patients undergoing open thoracoabdominal and descending thoracic aneurysm repair. J Thorac Cardiovasc Surg 147: 163-168, 2014.

8. Hiramori S, Soga Y, Kamioka N, et al. Clinical impact of the ankle-brachial index in patients undergoing successful percutaneous coronary intervention. Circ J 82: 1675-1681, 2018.

9. Sasaki M, Mitsutake Y, Ueno T, et al. Low ankle brachial index predicts poor outcomes including target lesion revascularization during the long-term follow up after drug-eluting stent implantation for coronary artery disease. J Cardiol 75: 250-254, 2020.

10. Nakahashi T, Tada H, Sakata K, et al. Impact of decreased anklebrachial index on 30-day bleeding complications and long-term mortality in patients with acute coronary syndrome after percutaneous coronary intervention. J Cardiol 74: 116-122, 2019.

11. Lee OH, Ko YG, Ahn CM, et al. Peripheral artery disease is associated with poor clinical outcome in patients with abdominal aortic aneurysm after endovascular aneurysm repair. Int $\mathrm{J}$ Cardiol 268: 208-213, 2018.

12. Gupta DK, Skali H, Claggett B, et al. Heart failure risk across the spectrum of ankle-brachial index: the ARIC study (Atherosclerosis Risk In Communities). JACC Heart Fail 2: 447-454, 2014.

13. Gerhard-Herman MD, Gornik HL, Barrett C, et al. 2016 AHA/ ACC guideline on the management of patients with lower extremity peripheral artery disease: a report of the American College of Cardiology/American Heart Association Task Force on Clinical Practice Guidelines. Circulation 135: e726-e779, 2017.

14. Thygesen K, Alpert JS, Jaffe AS, et al. Fourth universal definition of myocardial infarction (2018). J Am Coll Cardiol 72: 22312264, 2018.

15. Mizuno Y, Sakakura K, Yamamoto K, et al. Determinants of periprocedural myocardial infarction in current elective percutaneous coronary interventions. Int Heart J 61: 1121-1128, 2020.

16. Keisler B, Carter C. Abdominal aortic aneurysm. Am Fam Physician 91: 538-543, 2015.

17. Sawano M, Yamaji K, Kohsaka S, et al. Contemporary use and trends in percutaneous coronary intervention in Japan: an outline of the J-PCI registry. Cardiovasc Interv Ther 35: 218-226, 2020.

18. Yamamoto K, Sakakura K, Akashi N, et al. Novel acute myocardial infarction risk stratification (nARS) system reduces the length of hospitalization for acute myocardial infarction. Circ J 83: 10391046, 2019.

19. Yamamoto K, Sakakura K, Tsukui T, et al. Clinical factors associated with slow flow in left main coronary artery-acute coronary syndrome without cardiogenic shock. Cardiovasc Interv Ther 2020.

20. Taniguchi Y, Sakakura K, Adachi Y, et al. In-hospital outcomes of acute myocardial infarction with cardiogenic shock caused by right coronary artery occlusion vs. left coronary artery occlusion. Cardiovasc Interv Ther 33: 338-344, 2018.

21. Matsuo S, Imai E, Horio M, et al. Revised equations for estimated GFR from serum creatinine in Japan. Am J Kidney Dis 53: 982992, 2009.

22. Levey AS, Stevens LA, Schmid CH, et al. A new equation to estimate glomerular filtration rate. Ann Intern Med 150: 604-612, 2009.

23. IRON deficiency anaemia; report of a study group. World Health Organ Tech Rep Ser 182: 1-15, 1959.

24. Nutritional anaemias. Report of a WHO scientific group. World Health Organ Tech Rep Ser 405: 5-37, 1968.

25. Nishimura H, Miura T, Minamisawa $M$, et al. Prognostic value of ankle brachial index for future incident heart failure in patients 
without previous heart failure: data from the impressive predictive value of ankle brachial index for clinical long term outcome in patients with cardiovascular disease examined by ABI study. Heart Vessels 32: 295-302, 2017.

26. Chae CU, Pfeffer MA, Glynn RJ, Mitchell GF, Taylor JO, Hennekens $\mathrm{CH}$. Increased pulse pressure and risk of heart failure in the elderly. JAMA 281: 634-639, 1999.

27. Vaccarino V, Holford TR, Krumholz HM. Pulse pressure and risk for myocardial infarction and heart failure in the elderly. J Am Coll Cardiol 36: 130-138, 2000.

28. Yamazaki T, Goto S, Shigematsu H, et al. Prevalence, awareness and treatment of cardiovascular risk factors in patients at high risk of atherothrombosis in Japan. Circ J 71: 995-1003, 2007.

29. Miura T, Soga Y, Doijiri T, et al. Prevalence and clinical outcome of polyvascular atherosclerotic disease in patients undergoing coronary intervention. Circ J 77: 89-95, 2013.

30. Kim J, Lee JM, Park TK, et al. Long-term outcomes in patients undergoing percutaneous coronary intervention with or without preprocedural exercise stress test. J Korean Med Sci 35: e3, 2020.

31. Escaned J, Ryan N, Mejia-Renteria H, et al. Safety of the deferral of coronary revascularization on the basis of instantaneous wavefree ratio and fractional flow reserve measurements in stable coronary artery disease and acute coronary syndromes. JACC Cardiovasc Interv 11: 1437-1449, 2018.

32. Konishi $Y$, Ashikaga $T$, Sasaoka $T$, Kurihara K, Yoshikawa $S$, Isobe M. Comparison of outcomes after everolimus-eluting stent implantation in diabetic versus non-diabetic patients in the TokyoMD PCI study. J Cardiol 67: 241-247, 2016.

33. Brown CG, Kelen GD, Ashton JJ, Werman HA. The beta error and sample size determination in clinical trials in emergency medicine. Ann Emerg Med 16: 183-187, 1987.

The Internal Medicine is an Open Access journal distributed under the Creative Commons Attribution-NonCommercial-NoDerivatives 4.0 International License. To view the details of this license, please visit (https://creativecommons.org/licenses/ by-nc-nd/4.0/).

(C) 2021 The Japanese Society of Internal Medicine

Intern Med 60: 2733-2740, 2021 